\title{
El cesto de bienes y servicios territoriales: Un útil de diagnóstico aplicado a la Wilaya Argelina de Ain Témouchent
}

\author{
Ernesto GARCía ÁlVAREZ \\ Departamento de Geografía Humana. \\ Universidad Complutense de Madrid
Obdulia MONTESERÍN ABELLA
Departamento de Geografía Humana.
Universidad Complutense de Madrid omonteserin@ghis.ucm.es

\section{Silvia FREITAS CAETANO} \\ Departamento de Geografía Humana. \\ Universidad Complutense de Madrid

\section{Consuelo DEL CANTO FreSNO \\ Departamento de Geografía Humana. \\ Universidad Complutense de Madrid ccantofr@ghis.ucm.es}

\section{Susana RAMÍREZ GARCÍA}

Departamento de Geografía Humana.

Universidad Complutense de Madrid

Recibido: 6 de noviembre de 2013

Enviado a evaluar: 9 de enero de 2014

Aceptado: 30 de abril de 2014

\section{RESUMEN}

El modelo del cesto de bienes y servicios territoriales explica cómo y en qué condiciones la valorización conjunta de productos de calidad específicos de un territorio y los servicios asociados a ellos pueden contribuir al desarrollo territorial. El presente artículo parte de esta perspectiva y a través del caso de estudio de la wilaya de Ain Témouchent en Argelia prueba la aplicabilidad y validez de este modelo en un espacio rural de la orilla sur del mediterráneo. A partir del análisis de los diferentes elementos del modelo, una oferta específica de productos y servicios del territorio, una demanda vinculada al consumo de territorio y una combinación de bienes públicos y privados, se realiza un diagnóstico territorial que verifica ventajas e inconvenientes en la puesta en valor de los recursos y en los procesos necesarios para construir un cesto de bienes territoriales.

Palabras clave: Cesto de bienes, desarrollo territorial, gobernanza territorial, Ain-Témouchent. 


\title{
The territorial-based basket of goods and services:
}

\section{A useful tool of diagnosis applied to the Algerian Wilaya of Ain Témouchent}

\begin{abstract}
The model of the territorial-based basket of goods and services explains how valuing a group of specific quality products from a territory and the services related to them, could contribute to the territorial development. This article starts from this perspective and through the case study of the wilaya of Ain Témouchent in Algeria, it tests the applicability and validity of this model in a rural area on the southern shore of the Mediterranean. From the analysis of the different elements of the model, the specific offer of products and services of the territory, the demand generated by consumption linked to the idea of territory and the combination of public and private goods, we study the advantages and limitations in valuing local resources, as well as the necessary processes to configure a basket of territorial goods.
\end{abstract}

Key words: Basket of goods, territorial development, territorial governance, Ain-Témouchent.

Le panier de biens et services en tant qu'outil de diagnostic territorial: Le cas de la wilaya d'Ain-Témouchent (Algerie)

\section{RÉSUMÉ}

Le modèle du panier de biens et des services territorialisés explique comment et dans quelles conditions la mise en valeur en commun des produits de qualité liés à un territoire et des services qui leur sont associés peuvent contribuer au développement territorial. L'article part de cette perspective et, à travers l'étude de cas de la wilaya d'Ain-Temouchent en Algérie, teste l'applicabilité et la validité de cette approche dans un espace rural sur la rive sud de la Méditerranée. Un diagnostic territorial est réalisé à partir de l'analyse des différents éléments du modèle tels que l'offre spécifique des produits et des services du territoire, la demande liée à la consommation du territoire, et l'ensemble des biens publiques et privés, afin de vérifier les avantages et les inconvénients de la mise en valeur des ressources et des processus nécessaires pour construire un panier de biens territorialisés.

Mots clés: Panier de biens, développement territorial, gouvernance territorial, Ain Témouchent.

\section{INTRODUCCIÓN}

Este artículo presenta parte de los resultados obtenidos en los proyectos de investigación desarrollados a lo largo de los últimos nueve años por el Grupo de Investigación Complutense "Geografía para la Cooperación y el Desarrollo Territorial: una aplicación al ámbito mediterráneo" en la wilaya de Ain Témouchent (Argelia), en los que se han analizado y ensayado diversos enfoques y estrategias orientados a promover el desarrollo territorial ${ }^{1}$.

${ }^{1}$ Savoir-faire local et partenariat hispano-algérien dans la Méditerranée (2003-2004, UE, programa EuroMed); Formación a la carta para vitivinicultores argelinos (2005-2006, financiado por la AECID); Puesta en valor del saber hacer local vitivinícola de la wilaya de Ain Témouchent (2007-2008, financiado por la AECID) y Diversificación y dinamización de la viticultura de Ain Témouchent (2009-2011, financiado por la AECID). 
El interés del estudio se centra en el papel que pueden desempeñar los productos y servicios locales en el desarrollo territorial de un espacio rural y eminentemente agrícola como es la wilaya de Ain Témouchent, para ello se hace uso de la metodología del cesto de bienes y servicios territoriales diseñada por el equipo dirigido por Mollard y Pecqueur (Mollard, 2001; Pecqueur (2001); Hirczak et al., 2008)

El enfoque del "panier de biens" o cesto de bienes, parte de la idea de que en un mercado fuertemente competitivo, como es el de la industria agroalimentaria, una solución para las pequeñas empresas, que no pueden apoyarse en el tamaño para abaratar costes, es diferenciar sus productos haciendo uso de las competencias específicas no imitables y de los recursos originales. A partir de esta idea, el 'cesto de bienes' intenta explicar cómo, y bajo qué condiciones, la valorización conjunta de productos de calidad específicos de un territorio y de los servicios asociados a ellos puede contribuir al desarrollo territorial, proponiendo para ello "un método de investigación que pretende ayudar a los agentes de un territorio a evaluar el potencial de dicha valorización conjunta, y la posibilidad de definir, a partir de esto, una estrategia de desarrollo territorial" (Hirczak y Moalla, 2010)

Se ha considerado útil probar la posible validez de este enfoque en un territorio de la orilla sur del mediterráneo, que dista de las condiciones de organización, bienestar y desarrollo vigentes en los espacios europeos en los que se han llevado a cabo la mayoría de los estudios realizados hasta el momento. Interesa, sobre todo, analizar con los agentes locales en qué medida se cumplen en un espacio como Ain Témouchent las condiciones que el modelo determina como necesarias para que se produzcan dinámicas reales de desarrollo territorial, y proponer medidas que promuevan la emergencia y continuidad de dichas condiciones.

El trabajo de investigación se plantea analizar algunas de las dinámicas que animan un territorio de fuerte tradición agrícola y vitivinícola como es Ain Témouchent: identificar los problemas a los que se enfrentan los productores locales ante la obligada apertura de las economías motivada por la globalización, y determinar cómo resolverlos mediante la activación de sus recursos territoriales. En este segundo aspecto interesa abordar la puesta en marcha de mecanismos novedosos de coordinación y cooperación entre actores, estableciendo estrategias de desarrollo rural sostenible que relacionen agroindustria rural, territorio y acción colectiva.

El artículo se inicia con una revisión de las que se consideran las principales aportaciones del enfoque cesto de bienes al estudio del desarrollo territorial sostenible en espacios rurales, en concreto al diagnóstico territorial. A continuación se presenta el espacio en el que se ha llevado a cabo la investigación, a través de su ubicación geográfica y algunos datos que permiten hacerse una idea de conjunto. El siguiente apartado se centra en la aplicación de las herramientas de diagnóstico establecidas por el modelo del cesto de bienes. En este epígrafe se exponen el trabajo realizado, los datos obtenidos y los resultados de la aplicación del método elegido. Por último se presentan las conclusiones obtenidas sobre el espacio estudiado, así como una valoración de las posibilidades de la metodología empleada. 


\section{EL MODELO DEL CESTO DE BIENES Y LA DIAGNÓSIS TERRITORIAL}

\subsection{DESARROLLO TERRITORIAL Y CESTO DE BIENES}

Existe abundante bibliografía sobre el desarrollo territorial rural, tanto en el ámbito académico como en el de las políticas públicas, en particular en referencia a las heterogeneidades y desigualdades espaciales que tienden a acentuarse en la medida que se profundiza el fenómeno de la globalización en los territorios.

En este contexto, algunos enfoques teóricos se centran en las potencialidades de la valorización de los recursos locales, y la cualificación del espacio rural, a través de la promoción de dinámicas focalizadas en el establecimiento de sinergias que pueden surgir, espontáneamente o a partir de procesos de animación, del funcionamiento de las pequeñas empresas. Especialmente en lo que se refiere a productos agroalimentarios y tradicionales, estas empresas huyen de la competitividad por precio y basan sus estrategias en valores más difícilmente abordados por grandes empresas, como la cultura y la tradición (saber-hacer), la vinculación con un territorio (anclaje territorial), o como pueden ser las características de un producto que se relacionan con las singularidades geofísicas y climáticas de un territorio, esto es, diferencian sus productos haciendo uso de las competencias específicas no imitables y de los recursos originales.

En esta línea se encuentran las propuestas de los Sistemas Productivos Locales (SPL) (Courlet, 1994; Alonso y Méndez, 2000), su aplicación al sector agroalimentario, los Sistemas Agroalimentarios Localizados (SIAL) (Muchnik y Sautier, 1998), y los conceptos desarrollados por Mollard y Pecqueur de cesto de bienes y servicios territoriales (Mollard y Pecqueur, 2003), que serían la evolución de los conceptos anteriores en la medida que representan la idea de sistemas de tipo SPL y SIAL especializados en diferentes sectores, pero que se relacionan entre sí, posibilitando un beneficio territorial más generalizado y completo,; se trata, en este caso, de una modalidad de construcción territorial en torno a la calidad.

Según Ramírez y Del Canto (2008) "El conjunto de relaciones que se crean en torno a la valorización de los productos agroalimentarios localizados favorece la cualificación del espacio rural. A este respecto, Mollard (2001) afirma que "los productos agroalimentarios de calidad y labelizados poseen a la vez una calidad intrínseca ligada en particular al pliego de condiciones y al cuidado particular que comporta su producción (lo mismo que para los productos industriales) pero también una calidad de imagen y de origen construida por los agentes y su historia. La combinación de los dos tipos produce una Renta de Calidad Territorial" Aspectos todos ellos emparentados con el denominado "cesto de bienes y servicios territorializados", cuando en un territorio no es un solo producto agroalimentario, sino varios los que generan una renta territorial de calidad, y donde es el territorio mismo el que se vende."

Se parte, por tanto, de la idea de que una dinámica territorial en la que se establece un cesto de bienes y servicios, si su funcionamiento es el adecuado, llega a producir 
lo que se ha denominado Renta de Calidad Territorial, relacionada con los beneficios que puede traer la diferenciación de un producto o servicio por su anclaje territorial. La Renta de Calidad Territorial sería "un excedente con relación a la remuneración de los factores de producción (salarios, beneficios), que remuneran una parte de la producción y que no está espontáneamente vinculada a un actor o a un factor de producción preciso: se trata de una forma de internalización de los efectos externos a la producción stricto sensu, vinculados a nuevos recursos generalmente no mercantiles (factores gratuitos, bienes públicos, patrimonio...) y vinculados a la interacción entre la calidad de los productos y su anclaje territorial" (Cerdan et al., 2007, citando a Mollard).

\subsection{EL CESTO DE BIENES Y SERVICIOS TERRITORIALES}

La construcción teórica del cesto de bienes es fruto de las investigaciones llevadas a cabo desde hace más de quince años por un equipo de investigadores dirigidos por Amédée Mollar (Director de Investigación INRA-Université Pierre Mèndes France, Grenoble) y Bernard Pecqueur (Profesor del Institut de Géographie Alpine-Université Joseph Fourier, Grenoble), trabajos realizados dentro de un programa nacional de investigación que busca facilitar una cooperación activa entre investigación y desarrollo (Pour et Sur le Développement Regional -PSDR- en el subprograma realizado en Rhône-Alpes)

Como se ha expuesto en la introducción, "el enfoque cesto de bienes y de servicios es una propuesta de método de investigación que pretende ayudar a los agentes de un territorio a evaluar el potencial de dicha valorización conjunta, y la posibilidad de definir, a partir de esto, una estrategia de desarrollo territorial" (Hirczak y Moalla, 2010)

En sus análisis, Mollard y Pecqueur, identifican las potencialidades en términos productivos de los espacios rurales, con particular atención a los más frágiles, y ofrecen perspectivas desde la investigación-acción sobre las estrategias que permiten convertir la potencialidad de los recursos territoriales en un activo.

El modelo del cesto de bienes no es una representación simplificada de lo real ni un modelo normativo ideal que se deba preconizar, sino que se debe entender como la observación de regularidades y características que se repiten en situaciones no estrictamente idénticas y en las que es posible identificar tres elementos cuya presencia permiten la conformación del denominado 'cesto de bienes y de servicios' a partir del cual se pueden desarrollar estrategias sostenibles de desarrollo territorial. Estos tres componentes básicos son:

Una oferta específica de productos y servicios en un territorio, con un origen territorial común y unos atributos y características objetivas que los hace insustituibles por otros productos genéricos.

- Una demanda específica de esos productos, muy vinculada al consumo de territorio y por lo tanto poco 'elástica' en relación a los precios. 
Una combinación de bienes públicos y privados que rodean a los productos y los ponen en valor y que sólo son accesibles en el mismo territorio, reforzando la imagen y la reputación de calidad del territorio (Hirczak y Moalla, 2010)

El efecto de la concurrencia de estos tres elementos sobre el territorio vendría en parte determinado por las decisiones del consumidor, que, en un contexto de competencia imperfecta, estaría dispuesto a asumir precios más elevados por los productos en función de una fuerte componente subjetiva. Siguiendo a Hirczak et al. (2008) en la opción de compra del consumidor pueden entrar a formar parte los aspectos territoriales: «Bienes diferentes pueden estar ligados por una o varias características o atributos comunes, lo que les confiere una "identidad compartida" y una complementariedad que se encuentra, por ejemplo, en productos con el mismo origen o en servicios turísticos vinculados al mismo lugar. En este enfoque en términos de "bienes complejos territorializados", el consumidor desempeña un papel activo en la determinación de los atributos y busca, no un bien para el cual tendría una preferencia predefinida, sino el "servicio de base" que es susceptible de devolverle la integración de un conjunto de bienes complementarios (Lambin, 1993)»

Otro aspecto fundamental sería la interacción entre los actores públicos y privados, a este respecto Hirczak et al. (2008) señalan que "también hemos notado que a igual potencial de recursos, las oportunidades de desarrollo fueron mejor valorizadas en unas zonas que en otras, en particular donde los actores públicos y privados cooperaban entre ellos para mantener o reforzar estos procesos de valorización"

Se puede hablar, por tanto, del cesto de bienes como del espacio de encuentro entre una oferta variada de productos y servicios locales, en cuya definición entran a formar parte su historia, su tradición, su cultura (su anclaje territorial) y una demanda específica que reconoce, valora y está dispuesta a pagar estos atributos. Este espacio debe estar coordinado por la interacción entre actores públicos y privados.

\subsection{LA DIAGNOSIS TERRITORIAL A TRAVÉS DEL CESTO DE BIENES}

Según lo expuesto, para aquellos espacios en los que es evidente la ausencia de actividades económicas orientadas por la lógica globalizadora, la metodología propuesta por los autores del cesto de bienes parece adecuada para afrontar una diagnosis territorial. Este proceso de diagnóstico permite plantear el diseño de propuestas de investigación-acción para el desarrollo territorial fundadas en los recursos existentes y en las carencias detectadas.

La primera fase en el proceso de activación de un cesto de bienes consistiría en el citado diagnóstico territorial, para el que los autores (Hirczak y Moalla, 2010) proponen la realización del diagrama territorial cuyo fundamento se muestra en la figura 1. A partir de la construcción y análisis de este diagrama se obtendrían los datos necesarios para continuar con la propuesta metodológica del cesto de bienes, que avanzaría a través de diversas fases propositivas hacia la consecución de la gobernanza territorial. El ejercicio que se ha realizado en la investigación que se 
presenta se ha ceñido a la realización del diagnóstico territorial y la definición de líneas prioritarias de actuación a partir de éste.

Figura 1. Esquema para la construcción del diagrama territorial.

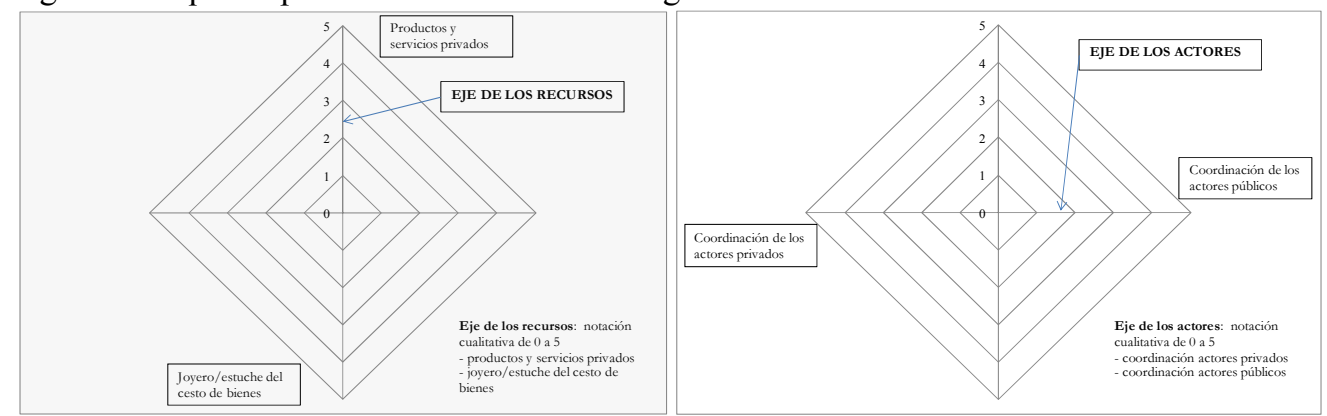

Fuente: Traducido de Hirczak y Moalla, 2010.

En el diagrama se ponen en combinación los tres componentes básicos del cesto de bienes citados con anterioridad y sus tres condiciones de perpetuación (Hirczak y Moalla, 2010):

- Coordinación entre actores privados

- Coordinaciones institucionales convergentes

- Coherencia entre acción privada y acción pública

Estos dos aspectos permiten calibrar la valorización de los recursos territoriales disponibles y la articulación de la gobernanza territorial, ítems cuya mejora conduciría a un desarrollo territorial duradero.

\subsection{LA ARTICULACIÓN ENTRE EL CESTO DE BIENES Y LA GOBERNANZA TERRITORIAL}

Los procesos de construcción y refuerzo de la gobernanza, sea local o territorial, pasan obligatoriamente por la configuración de redes en el territorio. La acción colectiva requiere que los actores locales sean capaces de construir relaciones entre ellos y con actores externos, lo que permitirá sacar el máximo beneficio económico, político, social y científico a toda la colectividad. Cuanta mayor diversidad de actores componen estas redes, más abundantes y variadas son las visiones y el conocimiento aportados en todo el proceso. Así,

Habitantes, científicos, especialistas en desarrollo y actores públicos pueden poseer información importante y un punto de vista determinado acerca de la preservación y la promoción de los recursos locales. Se trata de un saber compartido ya que se pueden considerar conocimientos especializados colectivos. El reconocimiento que han obtenido en el mercado los productos 
certificados con una IG evidencia la capacidad colectiva de definir y gestionar de manera eficaz la combinación de factores naturales y humanos. Además de definir el producto y su calidad determinada, la estrategia colectiva puede reforzar la reputación utilizada como instrumento estratégico para la comercialización o el desarrollo rural (Vandecandelaere et al., 2010)

La literatura, a través del "paradigma asociativo" (Cooke-Morgan, 1993, Méndez, 2002, Salom y Albertos, 2009), define tres dimensiones en cuanto al establecimiento $\mathrm{y}$ al papel de las redes sociales en formaciones espaciales productivas, como pueden ser las que conformen un cesto de bienes. La primera de estas dimensiones se refiere a las redes de colaboración empresarial, formadas sobre todo por actores económicos locales relacionados con producción, elaboración, servicios y comercialización, y su efectividad reside en la capacidad de obtener economías de escala, mayor aprendizaje colectivo y mejor aprovechamiento de los recursos. La segunda dimensión trata de las redes socioinstitucionales y empresariales para la innovación, en las que se establecen sinergias entre los recursos públicos y privados y una mayor capacidad de innovación. Y la última dimensión es la de las redes socioinstitucionales, que son las que promueven las nuevas formas de gobernanza en los territorios y la innovación institucional; son la expresión de un proyecto territorial común, resultado de un consenso entre los actores y están basadas en objetivos compartidos. Así, la expresión en el territorio de la buena gobernanza puede ser interpretada a través de las redes que se tejen.

Figura 2. Gobernanza territorial, cesto de bienes y renta.

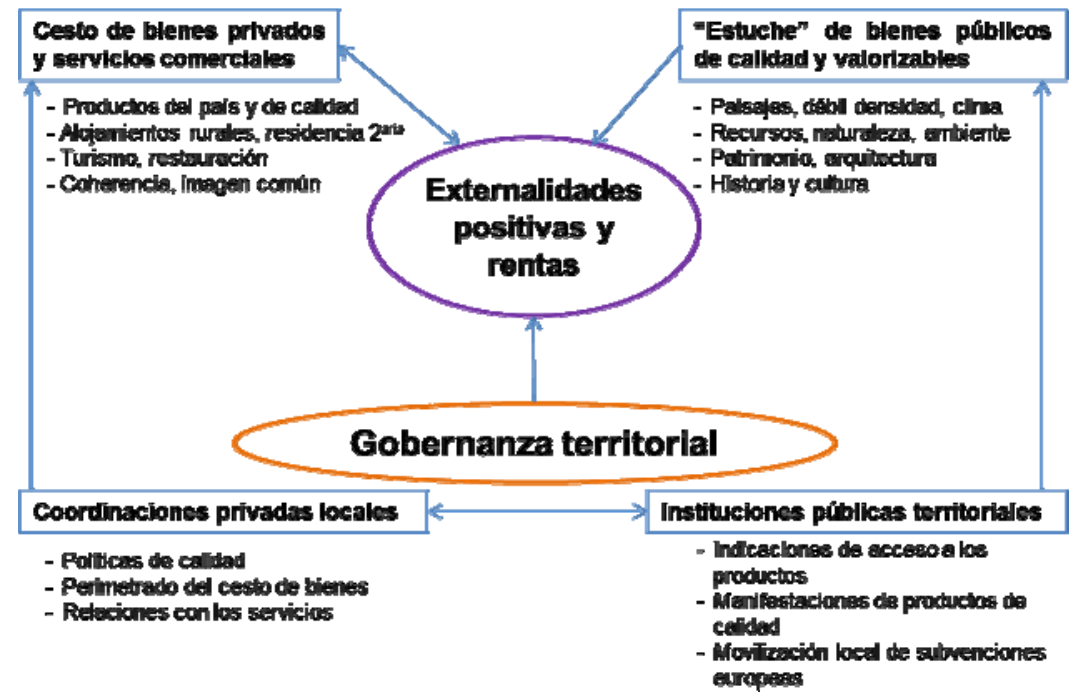

Fuente: Traducido de Hirczak y Moalla, 2010. 
En el siguiente gráfico (Figura 2) Hirczak y Moalla (2010) expresan la relación que se puede llegar a establecer entre el cesto de bienes, la gobernanza territorial y la renta, esta última como expresión numérica de las posibilidades de desarrollo que pueden surgir.

\section{LA WILAYA DE AIN TÉMOUCHENT}

El espacio en el que se ha realizado el estudio es la wilaya (provincia) de Ain Témouchent en Argelia. Se trata de un territorio ubicado en el noroeste del país, rodeado por las tres principales ciudades de la zona, Orán (segunda ciudad en importancia del país), la antigua ciudad imperial de Tlemcen y Sidi Bel Abbés, y a sólo $80 \mathrm{~km}$ de la frontera con Marruecos, lo que la convierte en un importante nudo de intercambio y comunicaciones en el noroeste del país. Está dividida administrativamente en 8 dairas o cantones que agrupan a 28 comunas o municipios. Su capital, Ain Témouchent, da nombre a la wilaya.

Figua 3. Comunas y Darías de la Wilaya de Ain Témouchent.

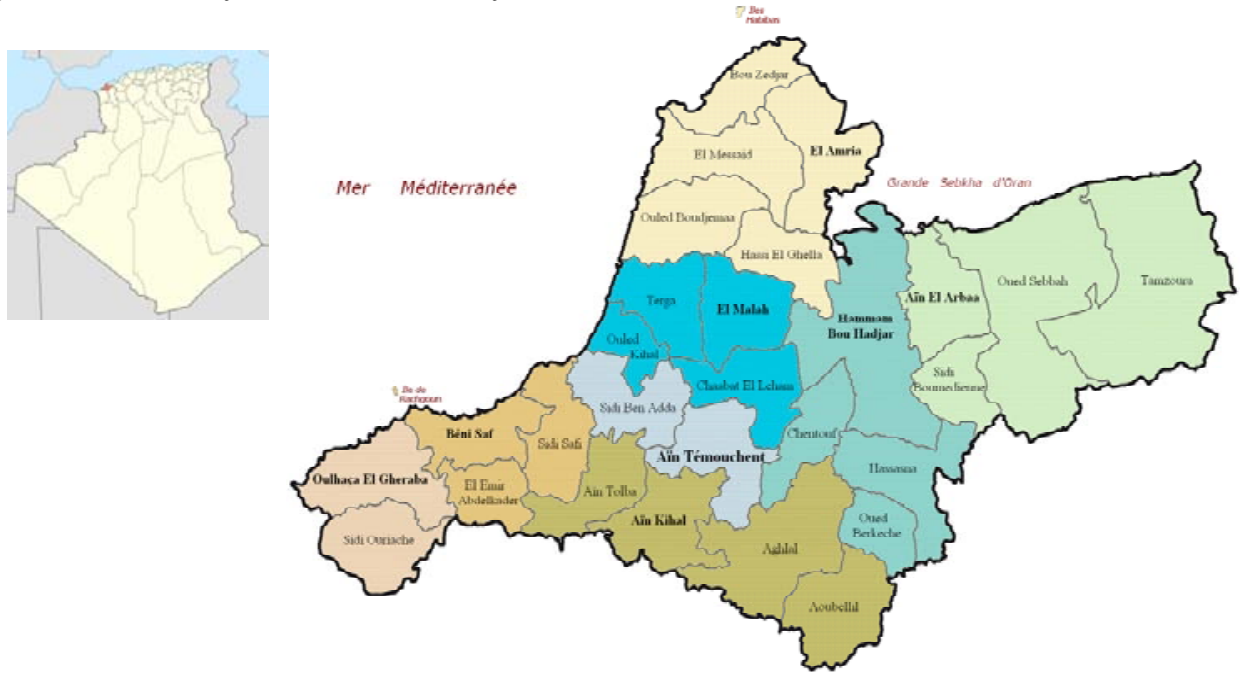

Fuente: Elaboración propia.

El último censo de 2008 fija la población de Ain Témouchent en 371.239 habitantes, con una densidad de $156 \mathrm{hab} / \mathrm{km}^{2}$. La mayoría de la población se concentra en las ciudades de Ain Témouchent y de Beni Saf y, en menor grado, en las comunas situadas a lo largo del importante eje de comunicación Oran-Tlemcen: El Malah, El Amria, Hassi El Ghella, Ain Tolba. 
Con una extensión de $2.376 .35 \mathrm{~km}^{2}, 80$ kilómetros de litoral, una altitud media de $250 \mathrm{~m}$. y un relieve moderadamente agreste, constituye un espacio de clara vocación agrícola, con una superficie agrícola útil de $180.184 \mathrm{Ha}^{2}(89 \%$ de la SAT, más del $70 \%$ del territorio), en su mayor parte de secano dada la elevada salinidad de gran parte del agua subterránea y de escorrentía que impide que se pueda utilizar como agua de riego (sólo dispone de $4.350 \mathrm{Ha}$. de regadío un 2,3 \% de la SAU). Los suelos, en gran parte de origen volcánico, son de excelente calidad y las condiciones climatológicas, propias de un clima mediterráneo suave, con una temperatura media anual de $17^{\circ} \mathrm{C}$ y unas precipitaciones débiles (entre 300 y $500 \mathrm{~mm}$ ) e irregulares, que determinan y limitan los cultivos.

En Ain Témouchent la viticultura tiene una fuerte tradición que se remonta a la época romana y que tuvo su máximo apogeo en la época de colonización francesa, durante la cual el viñedo y el vino dominaron de forma absoluta la economía y la organización de este territorio. Tras la descolonización y prácticamente hasta finales del siglo XX, la actividad vitivinícola vivió un fuerte y acelerado declive que se plasmó en el arranque de miles de hectáreas de viñedo y en el abandono de muchas de las bodegas e instalaciones de transformación vitivinícola que existían en la región. La sustitución de viñedo por cultivos cerealísticos provocó fuertes descensos en los rendimientos agrícolas y agravó los procesos erosivos y de desertificación que constituyen uno de los principales problemas medioambientales del noroeste argelino. En la actualidad el cultivo del viñedo continua jugando un papel especialmente importante dentro del tejido económico que articula este espacio y la wilaya tiene un importante peso específico dentro del sector vitivinícola argelino: la producción de vino de Ain Témouchent supone cerca del $40 \%$ de la producción total nacional con 13.156 ha de viñedo en 2011, un tercio de las cuales se dedican a uva de mesa.

Tabla 1. Ain Témouchent, evolución de la superficie de viñedo.

\begin{tabular}{|l|l|l|l|l|l|}
\hline \multicolumn{6}{|l|}{ Ain Témouchent - Evolución de la superficie de viñedo } \\
\hline Año & 1962 & 1998 & 2001 & 2004 & 2011 \\
\hline Ha viñedo & $60.000 \mathrm{Ha}$ & $8.600 \mathrm{Ha}$ & $18.040 \mathrm{Ha}$ & $24.468 \mathrm{Ha}$ & $13.156 \mathrm{Ha}$ \\
\hline
\end{tabular}

Fuente: DSA Ain Témouchent.

Tradicionalmente el viñedo ha convivido con otras producciones agrícolas, como los cereales ( 96.800 ha de cebada, trigo duro, trigo harinero, y avena), las leguminosas (garbanzos, habas, guisantes y alubias), y algunos cultivos forrajeros.

2 Todos los datos agrícolas citados han sido suministrados por la Direction du Service Agricole de Ain Témouchent y se refieren a la campaña 2011-2011. 
Existen también una cierta extensiones de terreno dedicadas a la arboricultura (con un fuerte peso del olivo) y a cultivos hortícolas.

Tabla 2. Potencial productivo de Ain Témouchent (campaña 2011-2012).

\begin{tabular}{|l|l|l|}
\hline Cultivo & Superficie (Ha) & \% SAU Total \\
\hline Barbecho & 22.800 & $12,48 \%$ \\
\hline Cereal & & \\
\hline Trigo duro & 34.800 & $19,04 \%$ \\
\hline Trigo harinero & 13.900 & $7,61 \%$ \\
\hline Cebada & 49.700 & $27,19 \%$ \\
\hline Avena & 3.500 & $1,92 \%$ \\
\hline Legumbres & & \\
\hline Garbanzos & 7.900 & $4,32 \%$ \\
\hline Habas & 2.900 & $1,59 \%$ \\
\hline Guisantes & 1.000 & $0,55 \%$ \\
\hline Alubias & 900 & $0,49 \%$ \\
\hline Forrajes & 8.000 & $4,38 \%$ \\
\hline Horticultura & 9.400 & $5,14 \%$ \\
\hline Cultivos perennes & & \\
\hline Arboricultura frutal & 3.335 & $1,82 \%$ \\
\hline Arboricultura de secano & 3.340 & $1,83 \%$ \\
\hline Oleicultura & 7.656 & $4,19 \%$ \\
\hline Viticultura & 476 & $0,26 \%$ \\
\hline Agrios & 13.156 & $7,20 \%$ \\
\hline & & \\
\hline
\end{tabular}

Fuente: DSA Ain Témouchent. 
En lo que se refiere a producción animal, la cabaña ganadera de Ain Témouchent no tiene un peso especialmente significativo (1.165 cabezas de bovino, 136.000 de ovino y 12.800 de caprino). La apicultura tiene una pequeña presencia en la wilaya (8.000 colmenas). La industria agroalimentaria de transformación es muy escasa (bebidas gaseosas, confitería,...) excepto las 20 bodegas operativas que quedan en la wilaya (en el momento de la independencia había más de 400 bodegas en funcionamiento en Ain Témouchent), propiedad de la cooperativa Viticoop, pero que son explotadas en su mayoría por las dos grandes compañía que dominan el sector vitícola argelino: GCO y ONCV.

La actividad pesquera tiene también su importancia en la región: Beni Saf, la segunda población de la wilaya, es uno de los más importantes puertos pesqueros de Argelia, con un volumen de capturas en 2007 de $31.061 \mathrm{Tm}$ mayoritariamente de pescado azul: sardina, chicharro y boga.

La industria está muy poco desarrollada y la escasa que existe predomina la fabricación de materiales de construcción (puzolana, tuba volcánica, piedra tallada). Las dos principales instalaciones industriales de la wilaya son la cementera SCIBS, ubicada en Beni Saf, y la fábrica de detergentes HENKEL, instalada en Ain Témouchent.

Otras actividades como la artesanía son marginales. En la wilaya están censados 126 artesanos tradicionales que trabajan en ramas como la cerámica, los tapices y la elaboración de prendas de lana, la cestería, o la confección de vestidos tradicionales.

Con respecto al sector servicios, en la estructura económica de la wilaya comienza a tener un peso importante la actividad turística, concentrada en las más de 30 playas aún no masificadas existentes en la costa y a algunas estaciones termales como la de Hamman Bouhadjard. Se trata sobre todo de un turismo nacional y de proximidad, algo explicable teniendo en cuenta el escaso desarrollo del turismo argelino internacional como consecuencia del cierre del país durante décadas al turismo internacional y la escasez y baja calidad de infraestructuras de acogida y alojamiento.

En lo que se refiere a otros servicios y actividades hay que tener presente que el peso de la economía informal es muy importante en Argelia y también lógicamente en Ain Témouchent, especialmente entre la población más joven que se dedica en buena parte a un comercio informal y callejero de menudeo. Siempre es difícil disponer de datos reales, pero según estimaciones del FMI, (FMI, 2013) realizados a partir de datos proporcionados por la Agencia Nacional de Estadística Argelina (ONS), la economía informal ha aumentado de manera considerable en la última década, de manera que en 2010 , un $45 \%$ del empleo total se englobaba dentro de la categoría de empleo informal. El mismo informe indica que la tasa de dinero en efectivo en circulación en Argelia está entre las más elevadas del mundo, lo que constituye un indicador que pone de relieve el insuficiente desarrollo del sector financiero, pero también revela que una parte sustancial de las transacciones comerciales se realizan fuera de los sistemas formales de pago y da una idea de la importancia de las actividades desregularizadas en la economía argelina. 


\section{LOS PLANEAMIENTOS DEL CESTO DE BIENES UTILIZADOS COMO HERRAMIENTA DE DIAGNÓSIS TERRITORIAL EN LA WILAYA DE AIN TÉMOUCHENT}

Durante los nueve años en los que el Grupo de Investigación ha trabajado en Argelia en proyectos centrados en la vitivinicultura de Ain Témouchent, se han realizado estudios de diagnóstico del sector, se han buscado soluciones para las problemáticas detectadas y se han puesto en práctica en colaboración con expertos técnicos en la materia.

El intenso trabajo desarrollado sobre el terreno, en estrecha cooperación con los actores locales, ha permitido obtener un conocimiento directo de las condiciones socioeconómicas de la región a través de la observación participante. Las dificultades que experimenta en su progreso el sector, en gran medida motivadas por la ausencia de coordinación entre actores privados y públicos y de gobernanza territorial han motivado la oportunidad de afrontar un ensayo de diagnóstico territorial desde la óptica del cesto de bienes.

Para ello ha sido necesario completar las investigaciones anteriores con la recopilación de datos estadísticos y con la realización de entrevistas abiertas y semiestructuradas y grupos de discusión, orientadas a recabar la opinión de los actores locales acerca de las problemáticas y expectativas de la wilaya y de las dinámicas establecidas entre los actores y las instituciones. En esta línea las temáticas afrontadas en las entrevistas han sido: cultura local, problemáticas de los productos tradicionales, receptividad hacia la diversificación de productos, percepción acerca del mercado consumidor, visión de futuro, representación y apoyo a nivel político y administrativo. Las entrevistas se han llevado a cabo con actores institucionales de la agricultura y el turismo y con actores privados del sector agrícola.

\subsection{LOS ELEMENTOS DEL CESTO DE BIENES}

\subsubsection{LA OFERTA}

Una oferta específica de productos y servicios en un territorio, con un origen territorial común y unos atributos y características objetivas que los hace insustituibles por otros productos genéricos.

Existe un elemento, altamente valorado, que todos los actores entrevistados coinciden en definir como la esencia de la wilaya: el viñedo. La importancia de este cultivo en el pasado y su pervivencia hasta la actualidad otorgan al territorio una singularidad y un carácter especial ligado al paisaje de viñedo. Un paisaje vulnerable $\mathrm{y}$ en claro retroceso pero que pervive en muchos espacios de Ain Témouchent y que es reconocido por los habitantes de la región como algo propio e identificativo. Un viñedo que se adecua perfectamente a las condiciones climáticas y edafológicas de la wilaya, fuertemente anclado en la memoria colectiva, que evoca los difíciles y a la vez prósperos tiempos de la colonización francesa en los que Ain Témouchent, la 
'perle de l'Oranie', era una de las wilayas más ricas y florecientes de Argelia como consecuencia de la explotación de más de 60.000 ha de viñedo y la existencia de más de 400 bodegas de las que salía buena parte del vino consumido en la época en Francia. Paradójicamente, este aprecio por el viñedo convive con un rechazo al vino, que se explica por motivos históricos, sociales y culturales y que es exhibido por una buena parte de la población, aunque se dé la contradicción de que la totalidad de la producción de vino de Argelia tiene como destino un mercado interior que además es deficitario.

La calidad potencial de los productos derivados de este viñedo es elevada, existiendo elementos muy valiosos como la Apellation d'Origine Contrôlée (AOC) Vino de Berkeche (Sahli, Z. y Mekersi, S., 2005). Por el momento son dos los productos provenientes del viñedo que dominan la economía del subsector: el vino y la uva de mesa. Otros subproductos potenciales: vinagre, zumos, pasas, mermeladas, etc. no tiene tradición productiva, aunque existe una cierta demanda y su potencial futuro es importante, como han puesto de manifiesto los estudios elaborados dentro del proyecto de cooperación "Puesta en valor del saber hacer local vitivinícola de la wilaya de Ain Temouchent" financiado por la AECID (Ait Habouche et al., 2009)

Junto al viñedo, como elemento que identifica y vertebra el territorio, aparecen otros productos agrícolas con un cierto anclaje territorial, e identificados por muchos de los actores como elementos identificativos del territorio. Los garbanzos de Ain Témouchent son apreciados a nivel nacional por su calidad. Otros productos como las aceitunas, higos o incluso una variedad de tomates local que está a punto de desaparecer, tienen también una fuerte especificidad pero no gozan del mismo reconocimiento interno y externo.

Vinculados al paisaje de la zona costera aparecen otra serie de productos y servicios con fuerte potencialidad. Los 80 kilómetros de costa ofrecen más de 30 playas, 20 de estas habilitadas para el baño,que reciben según datos de la Dirección de Turismo de la wilaya más de ocho millones de veraneantes al año (Figura 2).

Tabla 3. Infraestructura hotelera de playa de Ain Témouchent.

HOTELES

\begin{tabular}{llc} 
& & CAMAS \\
\hline Complexe Benchaábane & Terga plage & 160 \\
\hline Hotel Siga & Puits (Béni Saf) & 48 \\
Hotel Les Falaises & Sidi Boucif (Béni Saf) & 56 \\
Hotel El Nabil & Marmite (Béni Saf) & 420 \\
Hotel Madrid & Madrid (Béni Saf) & 96 \\
Hotel Le Dauphin & Rechgoun 1 (Béni Saf) & 170 \\
Hotel Syphax & Rechgoun2 (Oualhaca) & 260 \\
Hotel Bellaaredj Freres & & 48 \\
\hline
\end{tabular}

Fuente: Direction du Tourisme de la Wílya de AÍn Témouchent. 
El turismo ha crecido de manera importante en los últimos años pero ese crecimiento se ve frenado por la escasez y baja calidad de los servicios turísticos. A lo largo de la costa existen siete complejos hoteleros con una capacidad de 1.258 camas, muy por debajo de la demanda de los turistas en cantidad y calidad (Figura 4).

Como turismo de interior destaca la zona termal de larga tradición, Hammame Bouhdjar con treinta fuentes termales reconocidas cuya infraestructura de acogida ofrece 54 bungalows y 241 camas.

Figura 4. Potencialidades turísticas de Ain Témouchent.

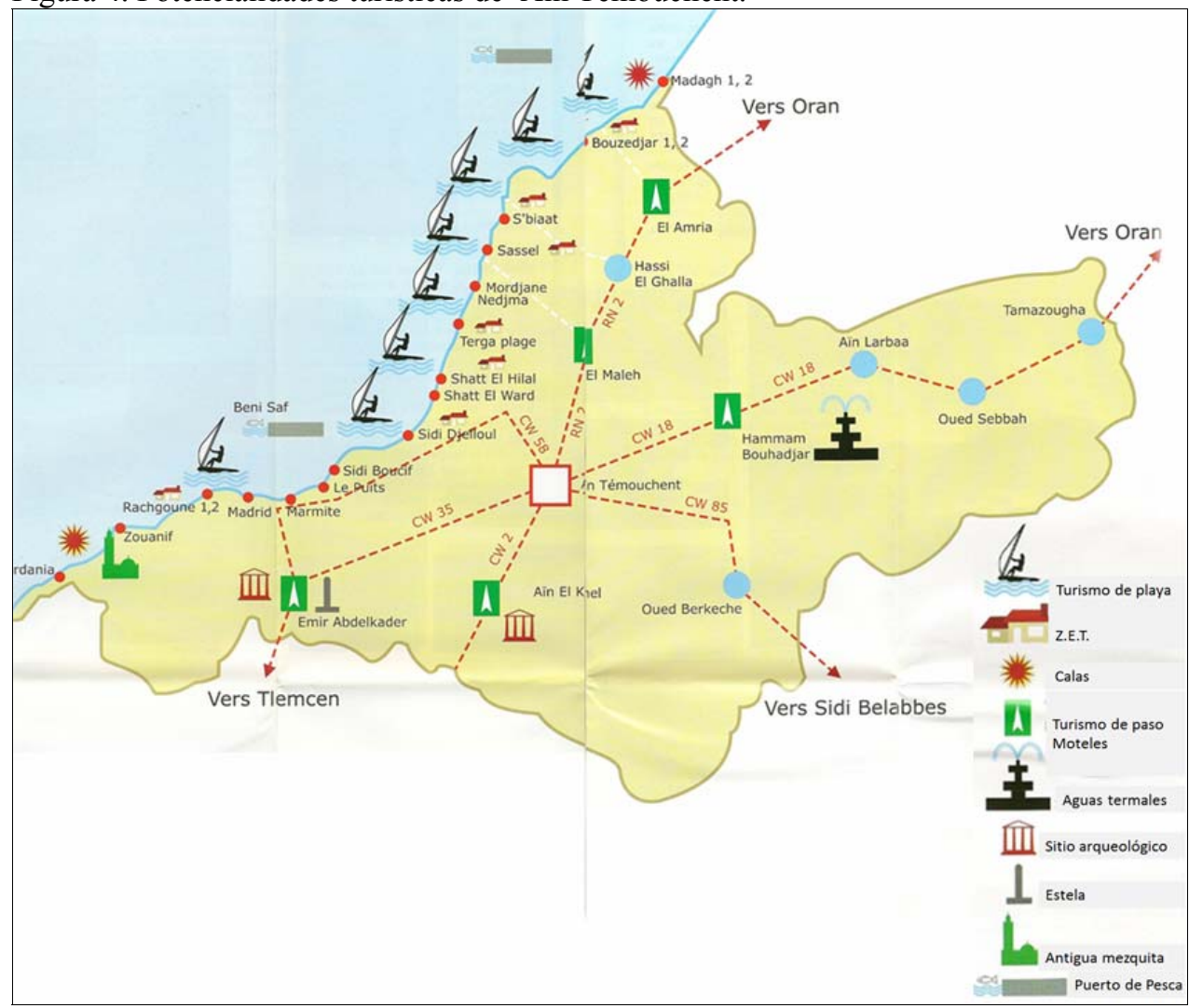

Fuente: Dirección de Turismo de la wilaya.

La Dirección de Turismo de la Wilaya de Ain Temouchent, busca, a través de la planificación de Zonas de Extensión Turistica, crear las condiciones para atraer inversiones en infraestructura turística, particularmente en las playas de más frecuencia. 
Tabla 4. Proyectos de Zonas de extensión turística (ZET)

\begin{tabular}{lc}
\hline ZET & hectareas \\
\hline Bouzedjar & 400 \\
\hline S'biaat & 180 \\
Sassel & 36 \\
Terga & 120 \\
Chatt El Hillal - Sidi Djelloul & 250 \\
Rechgoune & 50 \\
\hline
\end{tabular}

Fuente: Direction du Tourisme de la Wilaya d'Ain Témouchent.

Parece por lo tanto que en el territorio existen productos y servicios con la suficiente especificidad y vinculación territorial como para configurar lo que sería un cesto de bienes de Ain Témouchent: una serie de productos de la tierra (vino, uva, garbanzos, aceitunas, higos,...), que se completan con diversos servicios turísticos (playas, estaciones termales, pueblos y granjas coloniales, gastronomía, fiestas,...). Sin embargo, el modelo productivo de muchos de esos productos se encuadran más en un modelo genérico de carácter sectorial que en un modelo de producción local ligado a la calidad, a la tradición y al territorio, lo que dificulta otra de las condiciones expuestas por el modelo, la presencia de atributos y características objetivas que los hagan insustituibles por otros productos genéricos. También se observa que entre estos productos y servicios no se da la complementariedad que exige el modelo del cesto de bienes.

\subsubsection{LA DEMANDA}

Una demanda específica de esos productos, muy vinculada al consumo de territorio y por lo tanto poco 'elástica' en relación a los precios.

El análisis de la demanda de los productos identificados es sin duda el elemento más difícil del análisis realizado. Los circuitos de distribución y comercialización de Ain Témouchent distan mucho del modelo europeo, y en ellos predomina un microcomercio, muchas veces informal sobre el que es difícil obtener información y datos fiables.

El trabajo de campo y las encuestas realizadas a diferentes actores parecen indicar que en los hábitos de consumo de los productos con mayor vinculación al territorio priman factores como el coste, la presentación o la marca (aunque sea falsa) a la calidad o carácter local del mismo. La ausencia de indicaciones geográficas, no hace posible la identificación de los productos locales que en muchos casos el consumidor da por sentada, sobre todo en el caso de los mercadillos y zocos locales de productos agrícolas, algo que no siempre es la realidad ya que los productos pueden tener, y tienen, muy diversos orígenes. Se observa, no obstante, una demanda potencial hacia esos productos locales considerados por el consumidor conocedor de mayor calidad y 
atractivo. Pero hasta el presente no se ha identificado una demanda externa específica de productos locales, sobre todo vinculada al 'consumo del territorio' como podría ser la búsqueda por las formas y contextos tradicionales de consumo de dichos productos (modo de preparación, festividades, local de consumo, paisajes vinculados, etc.)

Por otra parte, como se verá más adelante, la ausencia de un proyecto colectivo que sirva para poner en valor los recursos locales tradicionales, es una de las barreras que dificulta la creación de una demanda específica capaz de mantener en activo estos recursos.

\subsubsection{LA COMBINACIÓN DE BIENES PÚBLICOS Y PRIVADOS}

Una combinación de bienes públicos y privados que rodean a los productos y los ponen en valor y que solo son accesibles en el mismo territorio, reforzando la imagen y la reputación de calidad del territorio

La imagen territorial de Ain Temouchent viene definida por los espacios agrarios, con un marcado peso del paisaje de viñedo, y por la línea de costa con sus más de treinta playasy los espacios naturales emblemáticos de la Reserva Natural de las islas Habibas y el islote de RechgouneEn lo que se refiere a patrimonio cultural, la herencia colonial está aún muy presente en Ain Témouchent con excelentes y bien conservados ejemplos de pueblos coloniales franceses como El Amria o el Maleh. Diseminadas por el territorio se encuentran numerosas mansiones y granjas coloniales de ambiente bucólico que podrían ser la base sobre la que desarrollar el turismo rural. Otros elementos culturales presentes en el territorio como la música y el folklore tradicional, los marabouts (morabitos o ermitas), las diversas fiestas y celebraciones religiosas, o la gastronomía (la paella de Beni Saf o los pasteles locales) constituyen recursos de interés aunque necesiten ser protegidos y revalorizados (Figura 2).

\subsection{EL DIAGNÓSTICO TERRITORIAL}

Para poder completar el diagnóstico territorial y la construcción del diagrama en los términos planteados por el modelo del cesto de bienes, se aborda, en primer lugar, el análisis de las tres condiciones de perpetuación del cesto.

\subsubsection{COORDINACIÓN ENTRE ACTORES PRIVADOS}

Los productos identificados anteriormente son reconocidos y consumidos localmente como tradicionales y típicos de la wilaya, pero no ha sido posible la construcción de un colectivo local cohesionado decidido a promover la imagen de esta oferta más allá de las fronteras de su propio territorio.

Según las opiniones recabadas en el trabajo de campo, la comunicación entre productores es débil en un entorno que parece caracterizarse por un fuerte individualismo y una escasa tendencia a cooperar fuera de la familia o clan. 
Sin embargo, se van constituyendo de forma espontánea grupos de interés común, productores que comienzan a colaborar y ponerse de acuerdo para solucionar problemas comunes, para demandar ciertos servicios o infraestructuras a la administración, o para buscar mejores mercados de salida a sus productos. Estos incipientes grupos de interés, podrían constituir el germen de futuras asociaciones de productores que articulasen y coordinasen a los diferentes actores del sector.

En cuanto a los servicios turísticos, la falta de estructuración del sector, hace que sea incapaz de coordinar las infraestructuras necesarias y de afrontar una adecuada divulgación de las atracciones locales.

\subsubsection{COORDINACIONES INSTITUCIONALES CONVERGENTES}

Las Instituciones Locales y los órganos que apoyan a la actividad agrícola en la región tienen, individualmente, programas y proyectos interesantes de fomento a la actividad, especialmente aquellos dirigidos hacia la formación de los trabajadores del campo, y hacia la continuidad de los jóvenes en esta labor. Sin embargo, no existe la costumbre de una actuación conjunta y coordinada entre las diferentes instituciones locales sectoriales (agricultura, transformación agroalimentaria, industria, turismo, etc.)

Por parte de la Administración el discurso oficial es que se trabaja a la escucha de los productores y consumidores y al servicio de los mismos, pero la realidad reconocida por ellos mismos habla de una política centralista y errática, muchas veces alejada de la realidad del territorio que ha conducido a los productores, en ocasiones, al borde del desastre.

La política seguida con el viñedo en la última década es un buen ejemplo de ello. En la primera mitad de la década anterior se puso en marcha un programa de recuperación del viñedo, basado en subvenciones a la plantación que no tuvo en cuenta la mejora de la calidad de las variedades plantadas, ni planteó salidas comerciales al producto final, el vino. Esta política se vio sustituida a finales de la década por otra en la que se gravó la producción y comercialización de vino, y se fomentó la producción de cereal y olivo, lo que se plasmó en dinámicas de arranque del viñedo plantado unos años antes. Entre ambas políticas, en 2005 se produjo la liberalización del sector productivo del vino. En este cambio, y ese mismo año, la ONCV, empresa estatal que hasta entonces tenía el monopolio de la transformación en vino y por lo tanto única compradora de uva para vinificación en la wilaya, rompió sus compromisos con los viticultores y la mayor parte de la cosecha de ese año se quedó sin comprador, con consecuencias dramáticas para los agricultores.

Vaivenes y decisiones políticas como las anteriores han provocado una profunda desconfianza con respecto a las políticas públicas y explican el escaso entusiasmo que despiertan entre los agricultores las diferentes propuestas de reconversión o modernización que llegan desde la Administración. 


\subsubsection{COHERENCIA ENTRE ACCIÓN PRIVADA Y ACCIÓN PÚBLICA}

Cuando se habla de coordinación y colaboración entre actores, el mayor déficit expresado por los productores es la falta de representatividad y eficacia de la cámara de agricultura, el principal organismo a través del cual se articula la comunicación entre productores y entre estos y la administración. Las acusaciones a dicho organismo se repiten: inmovilismo, falta de renovación, clientelismo, incapacidad,... con un mismo resultado, desconfianza y alejamiento de los productores del órgano que les debería coordinar y representar frente a la Administración.

El discurso inicial de muchos de los entrevistados es que la comunicación y cooperación entre productores y administración existe y es fluida. De manera similar, la comunicación y colaboración entre instituciones es, según la opinión 'oficial' de sus representantes, fluida y productiva. La realidad, sin embargo, que emana de las propias entrevistas es que la coordinación y colaboración entre los diferentes actores es muy escasa a todos los niveles: productores-productores, productoresAdministración, y Administración-Administración.

Los conflictos entre actores privados, la 'estanqueidad' de las relaciones entre estos y los actores públicos, y la falta de sinergia y coordinación entre todos ellos impide y bloquea estrategias de diferenciación y puesta en valor que permitan avanzar hacia la constitución de ese potencial cesto de bienes. Incluso si los actores privados colaboran a veces entre ellos, esta coordinación no se da con los actores públicos que además son múltiples y heterogéneos.

Tampoco existe la necesaria colaboración y coordinación entre los diferentes actores e instituciones para poner en valor los productos locales ni para promover su consumo. La apuesta por la calidad no es una prioridad ni para productores ni para la Administración, ocupados los primeros en incrementar la rentabilidad de sus productos con estrategias que priman la cantidad sobre la calidad, mientras que la Administración apuesta por desarrollar medidas y proyectos más centrados en políticas nacionales y sectoriales como es la seguridad alimentaria, que en estrategias locales orientadas a la valoración de los recursos locales y a la calidad.

Estas carencias en las relaciones se reflejan también en los aspectos más básicos, no existiendo participación popular en el diseño de las actuaciones institucionales para tratar de encontrar respuestas a las demandas específicas de los productores. Muchas de las demandas son, en realidad, bastante sencillas, como el suministro de energía eléctrica a algunas instalaciones productivas, sin embargo se ha detectado una ausencia de canales específicos que permitan que estas demandas sean conocidas por parte de las instituciones de apoyo.

\subsubsection{EL DIAGRAMA DE DIAGNÓSTICO TERRITORIAL}

Como se aprecia en el gráfico, existen recursos en la wilaya que podrían soportar la conformación de un cesto de bienes. Sin embargo, falta mucho trabajo en la estructuración de una oferta competente de los productos y servicios locales, y son 
prácticamente inexistentes las dinámicas de coordinación entre actores públicos, entre actores privados y entre ambos colectivos (ausencia de gobernanza territorial)

Figura 5. Diagrama de diagnóstico territorial de Ain Témouchent.

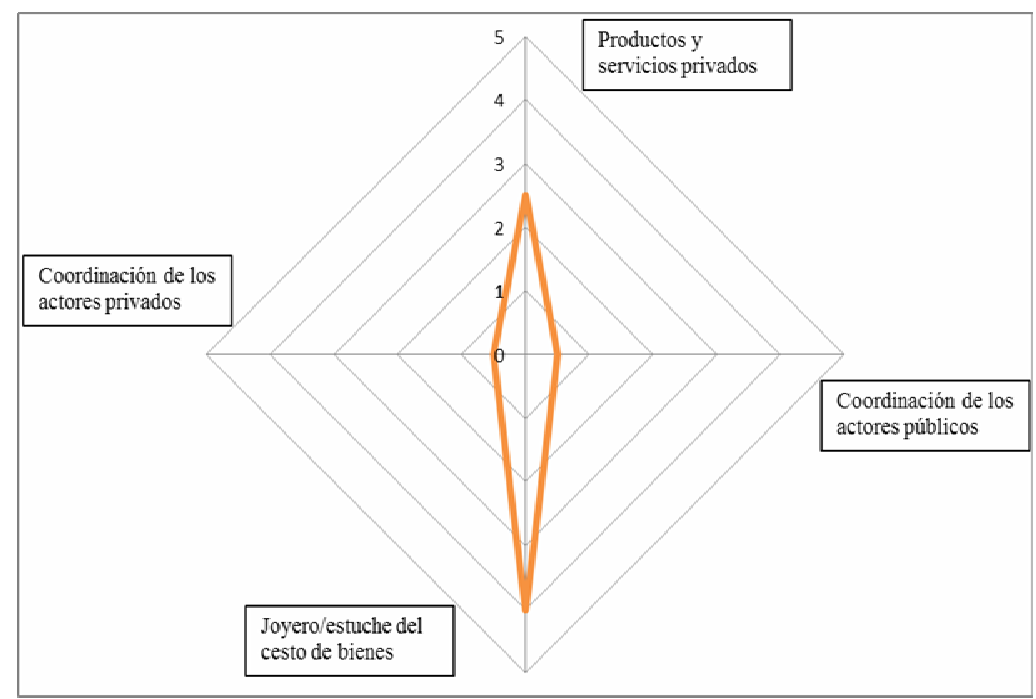

Fuente: Elaboración propia.

\subsubsection{VENTAJAS E INCONVENIENTES PARA LA CONFORMACIÓN DEL CESTO DE BIENES EN AIN TÉMOUCHENT}

El potencial de los productos y servicios territoriales es significativo, pero para que se produzca su puesta en valor de forma conjunta es necesario que los actores públicos y privados avengan a coordinarse y a colaborar. Se puede pensar que los actores tendrán un interés fuerte en cooperar para mejorar su posición y crear oportunidades de éxito, pero para que se establezca la cooperación se necesita previamente una proximidad entre actores, expresada por el desarrollo de una relación de confianza, y en Ain Témouchent esta relación de confianza por el momento no es evidente.

Como se ha visto en epígrafes anteriores, tejer una red sólida de relaciones parece una precondición para alcanzar una gobernanza territorial que tendría una repercusión muy positiva en el desarrollo de la región.

Existen iniciativas como la desarrollada por el proyecto de cooperación hispanoargelino en el marco del cual se ha desarrollado este trabajo de investigación, que apuestan, en colaboración con algunos actores locales, por avanzar en el anclaje territorial del vino y otros productos derivados del viñedo a través de la promoción de la etiqueta producto de Ain Témouchent. Como proyecto piloto se ha apoyado a la 
cooperativa local Viticoop en el desarrollo y elaboración de un vino de calidad de Ain Témouchent con buenos resultados. Los avances en este camino dependen sobre todo de la voluntad de los propios actores de continuar con la estrategia y de la capacidad de coordinación entre ellos y sus instituciones. Si la estrategia continúa, y da los frutos esperados, podría ejercer un efecto demostrativo sobre la dinámica de otros productos y servicios locales. La cooperación internacional tiene en este aspecto, a corto plazo, un papel tan importante como el que ha venido jugando en los proyectos hasta ahora desarrollados: actuar como agente animador del desarrollo territorial catalizando procesos de gobernanza territorial.

En lo que se refiere a los productos y servicios identificados con la suficiente especificidad y complementariedad como para entrar en un hipotético cesto de bienes, las acciones a proponer pasan por avanzar en dos líneas complementarias:

Mejorar la calidad, lo que exige cambios de mentalidad en todos los actores privados y públicos que permitan el paso de un modelo productivo en el que prima la cantidad y la competitividad en precios, a otro que prime la calidad e incremente el valor añadido.

- Profundizar en la especificidad y autenticidad de los productos a través del desarrollo de sistemas de control y certificación de indicación geográfica, y la promoción de modelos productivos anclados en la tradición, sostenibles económica y medioambientalmente. El papel de la administración en estos aspectos es fundamental, apoyando las iniciativas de coordinación entre productores para implantar sistemas de IG, desarrollando y aplicando un marco legal claro que proteja la especificidad y promueva las buenas prácticas medioambientales, y estableciendo los correspondientes sistemas de control.

Un primer paso en el desarrollo de estas líneas de trabajo, sería abrir un proceso participativo para la realización de un inventario colectivo de los bienes y servicios dotados de especificidad territorial, sobre el que dibujar las primeras actuaciones necesarias para su activación y puesta en valor.

Otro bloque de medidas, complementario al anterior, iría orientado a potenciar una demanda específica para esos productos, haciéndolos atractivos para aquellos consumidores dispuestos a pagar más a cambio de una serie de valores y características tanto tangibles (mayor calidad), como intangibles (exclusividad, vinculación con el territorio), capaces de generar una renta de calidad territorial (RQT) para los productores de esos bienes. El desarrollo de esta demanda puede, en función del contexto, ir ligado al desarrollo de marcas de calidad e indicaciones geográficas, al fortalecimiento de la imagen global del territorio y al desarrollo de un turismo sostenible y de calidad. El papel y coordinación de los diferentes actores pero especialmente los públicos es de nuevo fundamental, y exige trabajar para construir redes de cooperación dentro y fuera del territorio y promover y potenciar todo tipo de iniciativas privadas que ayuden a lograr los objetivos descritos. La toma de 
conciencia por parte de la población con respecto a la importancia de la buena imagen de su territorio y a la importancia y ventajas del turismo es fundamental para su recuperación. También lo es la estabilidad política y la ausencia de riesgos para visitantes y turistas, aunque estos elementos son difíciles de prever y controlar, y por supuesto la protección y promoción del patrimonio cultural y natural existente en Ain Témouchent.

\section{CONCLUSIONES}

El trabajo permite tener una visión de la realidad de Ain Témouchent y corroborar que está aún muy alejada del modelo de cesto de bienes existente en ciertas regiones rurales del norte de Mediterráneo. El panorama es el de un territorio con una imagen borrosa y débil, con unos productos ligados a ese territorio pero escasamente puestos en valor y con una complementariedad entre los productos y los servicios disponibles endeble. Un territorio en el que además hay importantes dificultades de coordinación y colaboración entre actores privados e instituciones.

Sin embargo, el análisis muestra también que existen los recursos potenciales para crear un cesto de bienes y que en torno a este modelo puede construirse una estrategia de desarrollo a medio y largo plazo que actúe sobre los factores que por ahora limitan la posible cristalización del modelo y potencie los recursos y aspectos más positivos hasta ahora encontrados. Esta estrategia debe fundarse en la reflexión sobre cómo y bajo qué condiciones la valorización conjunta de productos del terroir contribuye al desarrollo territorial.

El enfoque del cesto de bienes puede constituir una herramienta muy valiosa a la hora de determinar problemas y deficiencias y diseñar estrategias de desarrollo territorial a medio y largo plazo. Ain Témouchent busca aún su camino entre tradición y modernidad y está a tiempo de desarrollar dinámicas de desarrollo que, apoyadas en la tradición y especificidad del territorio, sean capaces de modernizar estructuras obsoletas e incrementar la renta y el bienestar sin renunciar a unas características e idiosincrasia propias. Lograrlo está sobre todo en manos de los actores públicos y privados que actualmente operan en el territorio y el éxito depende, más que de la existencia de recursos materiales o financieros, de la capacidad de esos actores para coordinarse y trabajar juntos por un objetivo claro y consensuado. La cooperación internacional puede actuar como catalizador en estos procesos, a través de proyectos de investigación -acción participativa que tiendan puentes entre los actores y aporten experiencias vividas por otros territorios. 


\section{BIBLIOGRAFÍA}

AIT HABOUCHE, A.; MERINO, S.; CONTRERAS, J., GARCÍA, E. Y MARTÍN, J. (2009): El sector vitivinícola de Ain Temouchent. Análisis de las posibles estrategias de diversificación. En: Diversificación y dinamización de la viticultura de Ain Temouchent. Asociación Tierras Sorianas del Cid, Soria.

ALONSO, J.L. Y MÉNDEZ, R. (coords.) (2000): Innovación, pequeña empresa y desarrollo local en España. Madrid, Civitas.

CERDAN, C., MARTINS DE SOUZA, M. C., Y FLORES, M. (2007): El patrimonio cultural como elemento estratégico para el desarrollo territorial - dos casos de la inmigración italiana en Brasil. Documento de Trabajo. Territorios con Identidad Cultural. Florianópolis, Brasil.

COOKE, P.; MORGAN, K. (1993): The network paradigm: new departures in corporate and regional development. En: Environment and Planning D: Society and Space 11(5)

COURLET, C. (1994): Les systèmes poductifs localisés. De quoi parie-t-on? En: Courlet, C y Soulage, B (Dir.): Industrie, territoires et politiques publiques. Paris. Éditions l'Harmattan, 13-32.

FMI (2013) Algeria Country Report, $n^{\circ} 13 / 47$, Febrero 2013. FMI, Washington.

HIRCZAK, M., Y MOALLA, M. (coord.) (2010): Le panier de biens et de services. Un noveau regard sur le développement des territoires. Rhône-Alpes PSDR/TPR

HIRCZAK, M., MOALLA, M., MOLLARD, A., PECQUEUR, B., RAMBONILAZA, M. Y VOLLET, D. (2008): Le modèle du panier de biens. Grille d'analyse et observations de terrain. Économie rurale, 308, 55-70.

MENDEZ, R. (2002): Innovación y desarrollo territorial: algunos debates teóricos recientes. En: Revista EURE, Santiago de Chile, vol. XXVIII, n ${ }^{\circ} 84$

MOLLARD, A. (2001): Qualité et développement territorial: une grille d'analyse théorique à partir de la rente. Économie rurale, 263, 16-34.

MOLLARD, A., PECQUEUR, B., HANUS, G., ROYBIN, D. Y GELIN, S. (2003): Valoriser les qualités spécifiques des territoires: et "panier de biens" des Baronnies. PSDR2, Territoires, acteurs et agricultures en Rhône-Alpes, Les résultats $\mathrm{n}^{\circ} 2$

MUCHNIK, J. Y SAUTIER, D. (1998): Systèmes agro-alimentaires localisés et construction de territoires. Proposition d'action thématique programmée. Paris, CIRAD.

LAMBIN,J. J. (1993). Marketing Estratégico. 2a ed. McGraw-Hill, París.

PECQUEUR, B. (2001). Qualité et développement territorial: l'hypothèse du panier de biens et de services territorialisés. Économie rurale, 261(1), 37-49.

RAMÍREZ GARCÍA, S. Y DEL CANTO FRESNO, C., (2008). Producciones agroalimentarias de calidad en el espacio rural madrileño. XI Coloquio Ibérico de Geografía: La perspectiva geográfica antes los retos de la sociedad y el medio ambiente en el contexto Ibérico. Textos completos de las ponencias y las comunicaciones. Universidad de Alcalá. 
SAHLI, Z.; MEKERSI, S. (2005). Etude de cas de Algérie. En: Ilbert, H. (2005): Produits du terroir mediterraneen: conditions d'emergence, d'efficacite et modes de gouvernance (PTM: CEE et MG). Rapport final. CIHEAM- IAMM. Montpellier

SALOM, J. Y ALBERTOS J. M., (2009). Redes socioinstitucionales, estrategias de innovación y desarrollo territorial en España. Colección Desarrollo Territorial. Serie Estudios y Documentos, 6. Universitat de València

VANDECANDELAERE, E.; ARFINI, F.; BELLETTI, G.; MARESCOTTI, A. (2010). Uniendo personas, territorios y productos; Guía para fomentar la calidad vinculada al origen y las indicaciones geográficas sostenibles. Strengthening International Research on Geographical Indications (SINER-GI). http://www.fao.org/docrep/013/i1760s/i1760s00.htm 